\title{
El papel de las tareas y los procesos en los resultados grupales: diferencias entre México y España
}

\author{
Amparo Osca*, Begoña Urien y María José Rodrigo
}

Universidad Nacional de Educación a Distancia (UNED) (Madrid, España)

\begin{abstract}
Resumen: Este trabajo analiza las diferencias en los procesos y resultados grupales en una empresa multinacional ubicada en España y México. La muestra está formada por 363 empleados: 121 españoles (que realizan tareas de fabricación) y 242 mexicanos (121 con tareas similares a las que realizan en España y 121 con tareas diferentes, prestación de servicios). Como se esperaba los resultados muestran que los empleados españoles perciben más positivamente todos los procesos (están más cohesionados y orientados a la tarea, se comunican mejor y manejan de forma eficaz sus conflictos), y resultados grupales (están más satisfechos y se consideran más eficaces) que los mexicanos. También en línea con lo esperado, se observan diferencias entre las muestras mexicanas a favor de la dedicada a prestar servicios, con tareas más enriquecidas. Por último, se estudia de forma exploratoria la influencia de los procesos en los resultados grupales en cada submuestra. Los resultados indican que la satisfacción se explica en las tres submuestras por los procesos relacionados con las interacciones sociales (comunicación y manejo eficaz de los conflictos), mientras que, para explicar la eficacia hay que atender a las diferencias entre países.

Palabras clave: Grupos de trabajo; México; España; procesos grupales; eficacia grupal.
\end{abstract}

\section{Introducción}

La globalización de las empresas generaliza los procesos de fabricación y de gestión desarrollados en contextos industrializados, en demasiadas ocasiones, sin poner a prueba su eficacia. Sin embargo, y como se ha señalado, no hay soluciones universales en la gestión y dirección del personal y, el éxito de determinadas prácticas depende del ajuste entre las creencias y los valores inherentes a los modelos que se quieren introducir y los de los grupos y personas implicados en los cambios (Robert, Probst, Martocchio, Drasgow y Lawler, 2000).

Este artículo analiza los procesos y resultados grupales en dos filiales de una misma empresa, ubicadas en México y España. Aunque no se dispone de datos acerca de los valores culturales de las muestras, el trabajo se aborda a la luz de la investigación transcultural y tomando el país como criterio para establecer las diferencias (p.e. Guarino y Feldman, 2005; Musitu y García, 2004; Ruiz, Sánchez y Menesini, 2002).

Destacar la escasez de estudios que comparen México y España desde una perspectiva similar. De la revisión bibliográfica realizada, el trabajo de Pavett y Morris (1995) analiza la influencia de la dirección participativa sobre la productividad en una empresa ubicada en cinco países, entre ellos México y España. Según sus resultados, el grado de

* Dirección para correspondencia [Correspondence address]: Amparo Osca. Dpto. Psicología Social y de las Organizaciones, Facultad de Psicología. UNED. C/ Juan del Rosal, 10. Madrid 28040 (España). E-mail:aosca@psi.uned.es
Title: The role of tasks and group on group outcomes: differences between Mexico and Spain.

Abstract: This paper analyzes the differences in group processes and outcomes in a multinational company. The total sample consists of $363 \mathrm{em}-$ ployees: 121 Spanish (engaged in production tasks) and 242 Mexican workers (121 engaged in production tasks and 121 performing services tasks to the production line). According to our results Spanish's perception of group processes (group cohesion, communication systems, orientation to the task and the effective management of conflict) and group results (satisfaction and effectiveness) is more positive than the perception of their Mexican counterparts. Besides, we have found several differences between the two Mexican samples, in group processes and group outputs in favor of those dedicated to providing services with more enriched tasks. Finally, we have studied the influence of the processes on the outputs from an exploratory perspective in each sample. The results show that satisfaction is explained by social interaction processes (communication and the effective management of conflict) in the three samples whereas to explain effectiveness we have to focus on the differences between the two countries.

Key words: Work groups; Mexico; Spain; group processes; group effectiveness.

participación difiere entre las filiales pero si esta participación es congruente con los valores culturales del país se consiguen niveles de productividad semejantes, independientemente de otras variables, como el estilo de dirección.

La mayor parte de los estudios transculturales parten de la clasificación de Hofstede $(1980,1993)$ y, más en concreto, de sus dimensiones culturales: individualismo (los sujetos esperan ser tratados por su valía personal y no por su pertenencia a grupos) y distancia de poder (los sujetos aceptan las diferencias entre las personas que ejercen la dirección y los subordinados). En su estudio, España se encuentra en el puesto once en individualismo y México en el dieciséis, y éste es además el país que presenta una mayor distancia de poder, mientras que España se sitúa en el décimo lugar.

También en el trabajo de Schwartz (1992) se observa que España y México muestran niveles claramente diferentes en dos de las dimensiones analizadas: España se encuentra en el tercer puesto en autonomía (los sujetos son independientes en su forma de pensar y en la toma de decisiones), frente al puesto diecisiete que ostenta México, y al contrario, es mucho menos conservadora (puesto diecisiete en conservadurismo: los sujetos se centran en el mantenimiento del status quo y ajustan su conducta a los usos sociales) que México (en la séptima posición).

Respecto a la influencia de los valores culturales en la conducta organizacional, las tesis en un primer momento apuntaban a que, en países colectivistas y con alta distancia de poder, las formas de gestión más participativas no darían buenos resultados. Uno de los primeros trabajos es el de Marrow (1967) sobre los problemas de una empresa puertorriqueña al implantar sistemas participativos. Años más tar- 
de, Pavett y Morris (1995) encuentran que, en países con alta distancia de poder, se prefieren estilos más autoritarios, mientras que en aquellos con puntuaciones más bajas se decantan por los más consultivos. En la misma línea, Pelled y Xin (1997), señalan que los mexicanos prefieren relaciones más jerárquicas entre jefes y empleados. Eylon y Au (1999) obtienen que los estudiantes de países con alta distancia de poder, trabajan peor y están menos satisfechos con aquellas tareas grupales que requieren colaboración e intercambio de información. En un estudio con directivos de 23 países, Smith, Dugan, Peterson y Leung (1998) observan que, en culturas con alta distancia de poder, hay un mayor rechazo a que los grupos desarrollen normas para manejar sus conflictos.

También se plantea que los colectivistas trabajan mejor en grupo pues están más confiados y motivados al sentirse miembros de un colectivo (Erez y Somech, 1996). Por ejemplo, Cox, Lobel y McLeod (1991) encuentran que, en culturas colectivistas, se da una mayor cooperación en las tareas grupales que en las individualistas. En algunos estudios se observa que los colectivistas trabajan mejor en grupo, con tareas interdependientes y compartiendo responsabilidades, $\mathrm{y}$ al contrario, los individualistas rinden mejor trabajando con metas y responsabilidad personal (Earley y Gibson, 1998). Sin embargo, Nicholl, Lane y Brechu (1999) cuando preguntan a los directivos qué destacan del trabajo en grupo, encuentran que los mexicanos señalan los aspectos socioemocionales y las buenas relaciones entre sus integrantes mientras que los estadounidenses prefieren los aspectos instrumentales y su contribución individual a la tarea.

Para Kirkman y Shapiro (1997) la clave está en que al introducir grupos de trabajo hay que considerar dos tipos de resistencias: la resistencia a trabajar en grupo y la resistencia a la auto-dirección, y proponen una clasificación de los países. En esta clasificación, México se encuentra en el escenario 3 ya que presenta una baja resistencia a trabajar en equipo (por su alto colectivismo) pero una mayor resistencia a la auto-dirección. Estos autores, ponen a prueba sus hipótesis con 461 equipos autodirigidos en cuatro países, y observan que aunque se cumplen parcialmente algunas de sus hipótesis, las relaciones son más complejas de lo que pensaban en un primer momento (Kirkman y Shapiro, 2001). Según sus datos, la resistencia a la introducción de equipos es una variable que media la relación entre los valores culturales y las actitudes laborales, y concluyen que hay que considerar otras variables que puedan mejorar las predicciones. En este sentido este trabajo analiza además de las diferencias entre países, las posibles diferencias debidas a las tareas.

Los estudios clásicos sobre tareas grupales y rendimiento se basaban en tareas relativamente simples, muy controladas y de corta duración, por lo que sus resultados son difícilmente generalizables a entornos laborales variados y complejos como los actuales (Tschan y Von Cranach, 1996). Por otro lado y, a pesar de la relevancia que se da a las tareas en los modelos de eficacia grupal (p.ej. Campion, Medsker y Higgs, 1993; Tannenbaum, Salas y Cannon-Bowers, 1996), la investigación no las ha analizado en profundidad (Guzzo y Shea, 1992; Ilgen, Mayor, Hollenbeck y Sego, 1993), aunque sí contempla aspectos como la variedad, la autonomía o la interdependencia (p.ej. Shaw, Duffy y Stara, 2000). Investigaciones más recientes sobre las características de las tareas, y concretamente sobre el modelo de Hackman y Oldham (1975), encuentran que se relacionan con los resultados grupales. Por ejemplo, el meta-análisis realizado por Steward (2006) sobre 93 estudios, confirma la relación significativa y positiva entre la autonomía grupal y el desempeño. Van Mierlo, Rutte, Kompier y Doorewaard (2005) encontraron relaciones significativas entre la autonomía y la satisfacción en una muestra de 76 equipos. Geister, Konradt, y Hertel, (2006), también observan que el feed-back sobre la tarea se relaciona positivamente con la satisfacción y el desempeño. El meta-análisis de Humphrey, Nahrgang y Morgeson (2007) (con 259 estudios) obtiene que las cinco características de la tarea de Hackman y Olham se vinculan positivamente con la satisfacción, y todas excepto la variedad explican la percepción de eficacia grupal.

Sin embargo algunos estudios cuestionan la validez de este modelo en culturas colectivistas. Por ejemplo Huang y Van de Vliert, (2003) muestran que las características del trabajo se relacionan con la satisfacción, pero sobre todo en países con culturas individualistas y con baja distancia de poder, mientras que Li, Li y Wang (2009) en equipos de empresas chinas, sí obtienen que las características se relacionan tanto con la satisfacción laboral como con el desempeño.

Siguiendo ambos planteamientos, las diferencias entre países y las diferencias entre tareas, este artículo analiza los procesos y resultados grupales en dos filiales de una misma empresa ubicadas en México y España. Para ello se han seleccionado tres submuestras, dos que realizan tareas similares en México y en España (mecanizado) y una en México con tareas diferentes (servicios). Esto permite analizar las diferencias entre países, y también entre tareas, y el papel de las tareas en la relación entre los procesos y resultados grupales.

En concreto se proponen las siguientes hipótesis.

Hipótesis 1: Los empleados mexicanos por su elevada distancia de poder, menor autonomía e individualismo y mayor conservadurismo, mostrarán más resistencia a trabajar en grupo que los empleados españoles, lo que se traducirá en una mayor dificultad en los procesos y resultados grupales.

Además en línea con la propuesta de Kirkman y Shapiro (2001) de considerar otras variables además de las culturales se analiza el papel de las tareas, ya que las diferencias entre los grupos mexicanos y españoles, puede depender también de las tareas que realizan.

Hipótesis 2: Los grupos mexicanos que tienen tareas más enriquecidas (grupos de servicios) mostrarán mejores procesos y resultados grupales que los grupos dedicados a tareas menos enriquecidas (grupos de mecanización).

Por último desde una perspectiva exploratoria, se analiza si los procesos influyen de forma diferente en los resultados grupales, en cada una de las tres submuestras consideradas. 


\section{Método}

\section{Muestra y Procedimiento}

Los datos se tomaron en las empresas durante la jornada laboral. Se recopilaron en la fase inicial de un proceso de consultoría en la que participaban miembros del equipo de investigación. Antes del pase definitivo, se presentó el cuestionario a los jefes de equipo y se pilotó con un grupo de 10 empleados para comprobar su comprensión y se realizaron las modificaciones oportunas. En ambos países la toma de datos se realizó en las mismas condiciones: antes de una sesión formativa y contestaron todos los empleados que trabajaban en equipo en ese momento. Aunque la recogida de información no coincide temporalmente, ambas filiales llevaban en torno a un año trabajando en equipo. Además en ese tiempo, no se dieron circunstancias especiales en ninguna de las filiales, que pudieran afectar a los resultados, por lo que se consideró que las comparaciones eran pertinentes.

Como la muestra española estaba formado por $121 \mathrm{em}-$ pleados de mecanizado, se seleccionó de la muestra mexicana más numerosa, dos submuestras con 121 empleados: una dedicada al mecanizado como la española, y otra a la prestación de servicios.

La muestra total consta de un total de 30 grupos (12 en España de mecanizado; 11 en México de mecanizado y 7 en México de servicios). El tamaño medio en la muestra española es de $12.58(D T=2.02)$, la muestra mexicana de mecanizado es 16.72 y de la de servicios $21.14(D T=3.31$ y $D T=$ 9.65 , respectivamente). Los grupos corresponden a los turnos de fabricación en el caso de las tareas de mecanizado y de servicio a las líneas de producción en el caso del grupo de servicios. El tamaño se corresponde con el número de efectivos necesarios para realizar el trabajo según el criterio de la empresa.

Para preservar el anonimato de los participantes, los datos sociodemográficos (edad y nivel de estudios) se pidieron en un formato de respuesta escalar que hacía más difícil la identificación. Respecto a la edad, el grupo más numeroso en las tres muestras es el que incluye a los empleados de 27 a 36 años (en España un 47.9\%; en México Servicios un $46.3 \%$ y en México Mecanizado 70.2\%), aunque, se encuentran diferencias significativas entre México y España $\left(\chi^{2}=31.109 ; p \leq .001\right)$, y entre México Mecanizado y México Servicios $\left(\chi^{2}=21.57 ; p \leq .001\right)$, de forma que los empleados mexicanos son más jóvenes que los españoles y, sobre todo, los de México Servicios, el 82.7\% oscila entre los 18 y los 36 años. En relación al nivel de estudios, sólo se encontraron diferencias significativas entre la muestra de España y las de México $\left(\chi^{2}=180.97 ; p \leq .001\right)$ ya que la mayor parte de los empleados mexicanos tienen el equivalente a BachillerSecundaria $(72.5 \%$ en México Mecanizado y $75 \%$ en México Servicios) mientras que en España no hay empleados con ese nivel y el grupo más numeroso es el de FPII (un 62.9\%). Respecto al sexo, en ambos países todos los empleados son hombres, algo común en este tipo de empresas.
Por último, los grupos se dividen en función de si realizan "tareas de mecanizado" (realización de diferentes partes del motor del automóvil) o "tareas de servicios" (dan apoyo cuando lo necesitan a otros grupos o líneas, p. ej. reparación de maquinaria, etc.). No obstante, en ambos casos se caracterizan porque: 1) están formados por empleados multicualificados y polivalentes, 2) el grupo asume tareas diferentes y variadas y además con significación y unidad, 3) las tareas se asignan al grupo y éste es el responsable del control de las mismas y, 4) al tratarse de la misma empresa, la coincidencia en las variables organizacionales y estructurales es muy elevada.

\section{Instrumentos}

Se evalúan dos tipos de variables los procesos y los resultados grupales. Se analizan cuatro procesos grupales: la orientación a la tarea, la cohesión grupal, los sistemas de comunicación y el manejo eficaz de conflictos. Como resultados se consideraron la satisfacción laboral y la eficacia percibida. Tanto los procesos como los resultados grupales se midieron con escalas Likert en las que la puntuación 1 se correspondía con el nivel más bajo y el 5 con el nivel más alto.

La orientación a la tarea se mide con una escala de 4 items, tomados del cuestionario de Normas Culturales de Kilman y Saxton (1983), y adaptado al castellano por Alonso, Osca y Palací (1996) (ítem ejemplo: “¿Cuánto esfuerzo dedican los miembros de su grupo a conseguir los objetivos grupales?"). La fiabilidad para la muestra total fue de .71. La cohesión grupal se evalúa con la escala de Urien (2001) diseñada a partir de los trabajos de Hyatt y Ruddy (1997) y Campion y col. (1993). Consta de 6 items y ofrece una fiabilidad de .74 . La escala pregunta por la calidad de la relación con otros miembros del grupo y la percepción de interdependencia en la realización de las tareas grupales (ítem ejemplo: “¿En su grupo hay un alto nivel de confianza entre sus miembros?”). Para evaluar los sistemas de comunicación grupal se utilizan 3 items diseñados para la ocasión y que preguntan por la facilidad con la que los miembros del grupo comparten información relevante para la realización del trabajo (ítem ejemplo: "Dentro de mi grupo la información fluye de manera espontánea y abierta”). La fiabilidad es de .74. El manejo eficaz de los conflictos se evalúa con una escala de 5 items inspirada en Fleishman y Zaccaro, (1992) y Hyatt y Ruddy, (1997). Esta escala pretende medir hasta qué punto el grupo posee una imagen positiva de las diferencias de opinión entre sus miembros y si se han establecido procedimientos para manejar sus discrepancias (ítem ejemplo: "¿En su grupo existen métodos adecuados para la resolución de los conflictos?”). Su fiabilidad es de .75. La escala de satisfacción se inspira en la de Spector (1985) y consta de 8 ítems que preguntan por el grado de satisfacción con diferentes aspectos del trabajo (ítem ejemplo: “¿Cuál es su grado de satisfacción con las relaciones con sus compañeros?"). La fiabilidad es de .74. Por último, la escala de eficacia percibida incluye 4 items que miden la eficiencia, la calidad, el grado de absentismo y la seguridad percibida en la consecución de los objetivos grupales (item ejemplo: “¿En 
qué medida su grupo de trabajo está consiguiendo los objetivos de calidad establecidos?"). La fiabilidad es de .74.

\section{Resultados}

Para evaluar la hipótesis 1 que analizaba las diferencias entre la empresa española y la mexicana en los procesos y resultados grupales se han realizado diferentes análisis de varianza (Tabla 1).

Tabla 1. Diferencias de medias en los procesos y resultados grupales entre México y España.

\begin{tabular}{|c|c|c|c|c|c|c|}
\hline \multicolumn{3}{|c|}{ MÉXICO } & \multicolumn{4}{|c|}{ ESPAÑA } \\
\hline & Media & D.T. & Media & D.T. & $F$ & $p$ \\
\hline PROCESOS & & & & & & \\
\hline Orient. a la tarea & 3.64 & .63 & 4.16 & .52 & 58.55 & .001 \\
\hline Sist. comunicación & 3.40 & .70 & 4.10 & .49 & 92.93 & .001 \\
\hline Cohesión grupal & 3.37 & .44 & 3.76 & .36 & 70.44 & .001 \\
\hline $\begin{array}{l}\text { Manejo eficaz de } \\
\text { conflictos }\end{array}$ & 3.08 & .64 & 4.10 & .49 & 71.59 & .001 \\
\hline RESULTADOS & & & & & & \\
\hline $\begin{array}{l}\text { Satisfacción labo- } \\
\text { ral }\end{array}$ & 3.61 & .50 & 4.10 & .44 & 79.23 & .001 \\
\hline Eficacia percibida & 3.16 & .77 & 3.77 & .59 & 58.21 & .001 \\
\hline
\end{tabular}

Como se esperaba, aparecieron diferencias significativas en todos los procesos grupales estudiados, de forma que la empresa española mostró valores más altos en los sistemas de comunicación $(F=92.93 ; p<.001)$, orientación a la tarea $(F=58.55 ; p<.001)$, manejo eficaz de los conflictos $(F=71.59$; $p<.001)$ y cohesión grupal $(F=70.44 ; p<.001)$. En la misma línea, los resultados grupales en España ofrecen valores superiores, pues los empleados están más satisfechos $(F=80.00 ; p<.001)$ y se perciben más eficaces $(F=58.21$; $p<.001)$.

Para comprobar el papel de las tareas en los procesos y resultados grupales (hipótesis 2) se han realizado también diferentes análisis de varianza. Como se puede comprobar en la Tabla 2 encuentran diferencias significativas entre la muestra de Mecanizado y la de Servicios en tres de los procesos: orientación a la tarea $(F=3.65 ; p<.05)$, sistemas de comunicación $(F=6.40 ; p<.01)$ y cohesión grupal $(F=4.52$; $p<.03$ ), como se esperaba a favor de los grupos dedicados a tareas de servicios más enriquecidas. En la misma línea se perciben más satisfechos $(F=24.05 ; p<.001)$ y más eficaces $(F=5.28 ; p<.02)$ (Tabla 2).

Además se ha analizado si los procesos influyen de forma diferente en los resultados grupales en cada una de las tres submuestras. Para ello se han realizado diferentes ecuaciones de regresión por pasos, sin especificar el orden en la entrada de las variables predictoras, con el objetivo de identificar los procesos más importantes en cada submuestra. Los análisis para cada submuestra toman como variables predictoras los procesos y como criterios los resultados.

En la Tabla 3, se puede comprobar que en los grupos españoles, la comunicación $(\beta=.69 ; p<.001)$ y el manejo eficaz de conflictos $(\beta=.21 ; p<.05)$ predicen significativamente la satisfacción laboral y, concretamente, la comunica- ción explica un 47\% de su varianza. En la muestra de México Servicios también es la comunicación el primer predictor significativo $(\beta=.47 ; p<.001)$, seguido de la orientación a la tarea $(\beta=.29 ; \phi<.05)$ y del manejo eficaz de conflictos $(\beta=$ $.20 ; p<.05)$. Por su parte, en la muestra mexicana de Mecanizado, los procesos grupales significativos son el manejo de conflictos $(\beta=.50 ; p<.001)$ y la cohesión $(\beta=.27 ; p<.01)$.

Tabla 2. Diferencias de medias en los procesos y resultados grupales entre las muestras de México (tareas de mecanizado y tareas de servicios).

\begin{tabular}{lcccccc}
\hline MÉXICO & \multicolumn{7}{c}{ TAREAS } & \multicolumn{7}{c}{ TAREAS } \\
& MECANIZADO & \multicolumn{2}{c}{ SERVICIOS } & & \\
\hline PROCESOS & Media & D.T. & Media & D.T. & F & P \\
Orient. a la tarea & 3.57 & .61 & 3.72 & .64 & 3.65 & .05 \\
Sist. comunicación & 3.29 & .68 & 3.52 & .70 & 6.40 & .01 \\
Cohesión grupal & 3.31 & .46 & 3.43 & .42 & 4.52 & .03 \\
Manejo eficaz de & 3.04 & .60 & 3.13 & .68 & 1.05 & .30 \\
conflictos & & & & & & \\
RESULTADOS & & & & & & \\
Satisfacción laboral & 3.46 & .49 & 3.77 & .47 & 24.05 & .001 \\
Eficacia percibida & 3.04 & .77 & 3.27 & .75 & 5.28 & .02 \\
\hline$*$ p $<.05$ & $* * \mathrm{p}<.01$ & $* * * \mathrm{p}<.001$ & & &
\end{tabular}

Tabla 3. Resultados de la ecuación de regresión de los procesos grupales sobre la satisfacción laboral en España y México (tareas de Mecanizado y tareas de Servicios)

\begin{tabular}{|c|c|c|c|c|}
\hline Variables Predictoras & $\beta$ & $\mathbf{F}$ & $\mathbf{R}$ & $\Delta$ de $\mathrm{R}^{2}$ \\
\hline \multicolumn{5}{|l|}{ España } \\
\hline \multicolumn{5}{|l|}{ Paso 1} \\
\hline Comunicación & $.69 * * *$ & $105.70^{* * *}$ & .47 & \\
\hline \multicolumn{5}{|l|}{ Paso 2} \\
\hline Comunicación & $.55^{* * *}$ & $58.10^{*}$ & .50 & $.02 *$ \\
\hline Manejo eficaz de conflictos & $.21 *$ & & & \\
\hline \multicolumn{5}{|l|}{ México Mecanizado } \\
\hline \multicolumn{5}{|l|}{ Paso 1} \\
\hline Manejo eficaz de conflictos & $.50 * * *$ & & & \\
\hline \multicolumn{5}{|l|}{ Paso 2} \\
\hline Manejo eficaz de conflictos & $.40 * * *$ & & & \\
\hline Orientación a la tarea & $.27 * *$ & $26.89 * * *$ & .30 & $.06 * *$ \\
\hline \multicolumn{5}{|l|}{ México Servicios } \\
\hline \multicolumn{5}{|l|}{ Paso 1} \\
\hline Comunicación & $.47 * * *$ & $31.99 * * *$ & .21 & \\
\hline \multicolumn{5}{|l|}{ Paso 2} \\
\hline Comunicación & $.32 * *$ & & & \\
\hline Orientación a la tarea & $.29 * *$ & $21.99 * * *$ & .27 & $.06 *$ \\
\hline
\end{tabular}

Respecto a la eficacia percibida (Tabla 4), en España el principal proceso grupal es la orientación a la tarea $(\beta=.42$; $p<.001)$, seguido de la cohesión y del manejo eficaz de los conflictos $(\beta=.26 ; p<.01)$. En la muestra de México Servicios la variable predictora de más peso es el manejo eficaz de los conflictos $(\beta=.49 ; p<.001) \mathrm{y}$, en segundo lugar, la orientación a la tarea $(\beta=.26 ; p<.001)$. Estos resultados coinciden con los de México de Mecanizado donde los procesos que mejor predicen la eficacia grupal son el manejo eficaz de los conflictos $(\beta=.52 ; p<.001)$ y la orientación a la tarea $(\beta=.25 ; p<.01)$. 
Tabla 4. Resultados de la ecuación de regresión de los procesos grupales sobre la eficacia percibida en España y México (tareas de Mecanizado y tareas de Servicios).

\begin{tabular}{|c|c|c|c|c|}
\hline Variables predictoras & $\beta$ & $\mathbf{F}$ & $\mathbf{R}$ & $\Delta$ de $\mathrm{R}^{2}$ \\
\hline \multicolumn{5}{|l|}{ España } \\
\hline \multicolumn{5}{|l|}{ Paso 1} \\
\hline Orientación a la tarea & $.42^{* * *}$ & $25.19^{* * *}$ & .17 & \\
\hline \multicolumn{5}{|l|}{ Paso 2} \\
\hline Orientación a la tarea & $.31 * * *$ & & & \\
\hline Cohesión & $.26 * *$ & $17.42^{* * *}$ & .22 & $.05^{* *}$ \\
\hline \multicolumn{5}{|l|}{ México Mecanizado } \\
\hline \multicolumn{5}{|l|}{ Paso 1} \\
\hline Manejo eficaz de conflictos & $.52 * * *$ & $43.46^{* * *}$ & .27 & \\
\hline \multicolumn{5}{|l|}{ Paso 2} \\
\hline Manejo eficaz de conflictos & $.37 * * *$ & & & \\
\hline Orientación a la tarea & $.25^{* *}$ & $26.56^{* * *}$ & .30 & $.04 * *$ \\
\hline \multicolumn{5}{|l|}{ México Servicios } \\
\hline \multicolumn{5}{|l|}{ Paso 1} \\
\hline Manejo eficaz de conflictos & $.49 * * *$ & $39.77 * * *$ & .24 & \\
\hline \multicolumn{5}{|l|}{ Paso 2} \\
\hline Manejo eficaz de conflictos & $.38^{* *}$ & & & \\
\hline Orientación a la tarea & $.26 * *$ & $24.15^{* * *}$ & .29 & $.05^{* *}$ \\
\hline
\end{tabular}

\section{Discusión}

Este trabajo ha analizado el papel de las tareas y los procesos en los resultados grupales en dos filiales de una empresa multinacional. Sin disponer de datos acerca de sus valores culturales, pero basándonos en la literatura transcultural, se han formulado una serie de hipótesis sobre las diferencias en los procesos y los resultados grupales en México y España. También se ha puesto a prueba la influencia del tipo de tareas que desempeñan los empleados y su papel en la percepción de la eficacia del sistema de trabajo en grupos.

Concretamente se planteó que la elevada distancia de poder, la menor autonomía y el mayor conservadurismo de los empleados mexicanos, podía llevarles a mostrar una mayor resistencia al trabajo en grupo, lo que se traduciría en una mayor dificultad en los procesos y resultados grupales (hipótesis 1). Confirmando esto se encuentran importantes diferencias a favor de los empleados españoles en todos los procesos y resultados grupales analizados. Esto va en la línea de la propuesta de Kirkman y Shapiro (1997), de que en los países más colectivistas y con mayor distancia de poder, como es el caso de México, la introducción de equipos puede resultar más complicada. Los procesos grupales analizados presentan más dificultades en los equipos mexicanos, cuyos empleados se muestran menos orientados a la tarea y con menor cohesión, y con más problemas de comunicación y dificultades para resolver sus conflictos. Lo mismo ocurre con los resultados grupales, ya que los mexicanos se manifiestan menos satisfechos con su trabajo y perciben que sus equipos son menos eficaces que los españoles.

Siguiendo la propuesta de Kirkman y Shapiro (2001) de analizar otros aspectos, además de las diferencias entre países, se ha estudiado el papel de las tareas (hipótesis 2) en la relación entre los procesos y resultados grupales. Como se esperaba, en la muestra de México aparecen diferencias importantes en función de las tareas que realizan los equipos, de forma que, aquellos con tareas más enriquecidas, es decir los dedicados a prestar servicios, siguiendo el planteamiento de Hackman y Oldham (1975), presentan una mayor cohesión, se comunican mejor y están más orientados a su trabajo, más satisfechos y se perciben más eficaces que los dedicados a tareas de mecanizado. Estos datos coinciden con la investigación que señala que el modelo de las características del puesto sigue siendo eficaz en países colectivistas y sus planteamientos son igualmente aplicables a otros contextos culturales (Li y col., 2009).

Por último, se analizaba de forma exploratoria, el papel de los procesos en los resultados grupales y la posibilidad de que se dieran discrepancias entre las tres submuestras. Los resultados, en línea con los anteriores, apuntan diferencias pero dependen, de la variable resultado. La satisfacción se explica por procesos vinculados a las relaciones sociales como la comunicación y el manejo eficaz de los conflictos. Concretamente, en los empleados españoles y los mexicanos dedicados a los servicios, la satisfacción depende fundamentalmente de una buena comunicación. En la misma línea, los grupos mexicanos con tareas de mecanizado, de mayor tamaño que los españoles, la satisfacción se explica porque existan mecanismos para manejar y resolver los conflictos de forma más adecuada.

Por su parte, la percepción de eficacia grupal se explica mejor diferenciando por país. En México, los procesos más importantes son el manejo eficaz de los conflictos y la orientación a la tarea, independiente de las tareas que se realicen, mientras que en España es estar orientado a los aspectos instrumentales del trabajo. Desde la perspectiva de los estudios transculturales, estos datos se pueden explicar por el mayor interés de los empleados mexicanos por la armonía en las relaciones entre los miembros de su grupo (Nicholl y col., 1999). Así, disponer de normas para manejar los conflictos se revela, en los equipos mexicanos como el mecanismo fundamental para explicar la percepción de eficacia grupal. Sin embargo, el mayor individualismo de los españoles les lleva a valorar los aspectos más instrumentales y fundamentalmente, que sus compañeros estén orientados a la realización de las tareas grupales.

En conjunto, y para resumir, se aprecian coincidencias en las razones por las que los miembros de los equipos se sienten satisfechos. En las tres muestras son procesos de carácter relacional, concretamente la comunicación y el manejo de conflictos. Sin embargo, en la percepción de eficacia hay diferencias entre países. En España se perciben más eficaces aquellos empleados más orientados al trabajo, y en México los que disponen de formas para gestionar sus conflictos.

Respecto a las limitaciones de este trabajo destacar que los términos transcultural y transnacional no son sinónimos (Drenth y Groenendijk, 1998), y que no todas las diferencias entre países pueden considerarse diferencias culturales y viceversa. De ahí que, en futuros trabajos, haya que recoger 
información sobre los valores culturales de cada submuestra, sobre todo si se tiene en cuenta que probablemente, ni los empleados españoles ni los mexicanos de nuestro estudio, sean representativos de los sujetos de sus países. Otra limitación está relacionada con las características de los empleados y el trabajo que realizan. Contar con información de operarios en situaciones reales, es muy interesante y difícil de conseguir, de ahí la ausencia de investigación en este colectivo, como se ha señalado recientemente (Strauch, 2010), no obstante parece fundamental contrastar los resultados con muestras diferentes y que realicen tareas con mayores exigencias cognitivas. Por último, convendría contar con información objetiva acerca del desempeño grupal, aunque nuestros resultados van en la línea de los de Newman y Nollen (1996) quienes encuentran que el rendimiento se relaciona con el ajuste entre los valores culturales y las prácticas organizativas del país.

Sin embargo, a pesar de las limitaciones señaladas creemos que este trabajo arroja luz sobre la introducción de equipos de trabajo en empresas multinacionales y las dificultades que pueden aparecer al exportar determinadas prácticas de gestión a terceros países. Desde una perspectiva aplicada, nuestros resultados ponen de manifiesto la necesidad de aproximaciones integradoras a la hora de implantar equipos. Así y además de considerar las variables incluidas en los modelos de eficacia grupal tradicionales, habrá que contem-

\section{Referencias}

Alonso, E., Osca, A. y Palací, F. (1996). El patrón ideal de la cultura organizacional. Revista de Psicología Social, 11(1), 33-46.

Aykan, Z. y Kanungo, R.N. (2001). Crosscultural Industrial and Organizational Psychology: A Critical Appraisal of the Field and the Future Directions. En N. Anderson, D.S. Ones, H.K. Sinangil y Ch. Viswesvaran (Eds.), Handbook of Industrial, Work and Organizational Psychology (pp. 385408). London: Sage Publications.

Bochner, S. y Hesketh, B. (1994). Power distance, individualism/collectivism, \& job-related attitudes in a culturally diverse work groups. Journal of Cross-Cultural Psychology, 24, 233-257.

Campion, M.A., Medsker, G.J. y Higgs, A. (1993). Relations between work group characteristics and effectiveness: Implicacions for designing effectiveness group. Personnel Psychology, 46, 823-850.

Cox, T.H., Lobel, S.A. y McLeod, P.L. (1991). Effects of ethnic group cultural differences on cooperative and competitive behavior on a group task. Academy of Management Journal, 34(4), 827-847.

Drenth, P.J.D. y Groenendijk, B. (1998) Organizational Psychology in a Cross Cultural Perspective. En P.J.D. Drenth, H. Thierry y C.J. de Wolf (Eds.), Organizational Psychology (pp. 133-154). East Sussex: Psychology Press Ltd.

Earley, P.C. y Gibson, C.B. (1998). Taking stock in our progress on individualism-collectivism: 100 years of solidarity and community. Journal of Management, 24(3), 265-304.

Earley, P.C. y Mosakowski, E. (1994). Experimental Internal Management Research. En B.J. Punnett y O. Shenkar (Eds.), Handbook of International Management Research (pp. 83-114). Cambridge, MA: Blackwell.

Erez, M. y Somech, A. (1996). Is group productivity loss the rule or the exception? Effects of culture and group-based motivation. Academy of Management Journal, 39(6), 1513-1537.

Eylon, D. y Au, K.Y. (1999). Exploring empowerment cross-cultural differences along the power distance dimension. International Journal of Intercultural Relations, 23(3), 373-385. plar también los valores culturales. Como se ha apuntado, la formación puede mejorar el desempeño grupal (Salas, Diaz, Klein, Burke, Stagj, Goodwin y Halpin, 2008) y reducir el posible efecto negativo de la cultura (Klein y Steel-Johson, 2007). En este sentido habrá que diseñar actividades de sensibilización que incrementen la aceptación de los nuevos sistemas de trabajo y reducir las resistencias a los cambios que se van a introducir. Como señalan Kirkman y Shapiro (1997) se deberá atender a: 1) la identificación de los valores culturales de los empleados para hacerlos más compatibles con el sistema de trabajo en grupo; 2) la adaptación de algunas características de los grupos para conseguir el mejor ajuste a los valores culturales de cada país; 3) el cambio de las estrategias en función de los esos valores, y, 4) la elección precisa los agentes de cambio, dedicando especial atención a aquellos equipos con: un tamaño reducido, tareas muy interdependientes y con miembros similares o de alto estatus, porque son los que muestran mayor resistencia a los cambios.

Para finalizar como señalaban Aykan y Kanungo (2001), es necesario reforzar dentro de la Psicología del Trabajo y de las Organizaciones una perspectiva transcultural que analice los modelos de los que partimos y su validez en contextos culturales diferentes, si no es así, se dejará de lado a muchos empleados cuyo protagonismo en nuestra sociedad es cada vez mayor.

Fleishman, E.A. y Zaccaro, S.J. (1992). Toward a taxonomy of team performance functions. En R.W. Swezey, y E. Salas (Eds.), Teams: Their training and performance (pp. 31-56). Westport, CT, US: Ablex Publishing.

Gladstein, D.L. (1984). Groups in context: A Model of Task Group Effectiveness. Administrative Science Quarterly, 29, 499-517.

Guarino, L. y Feldman, L. (2005). La diferencia de la sensibilidad emocional entre británicos y venezolanos. Psicothema, 17(4), 639-644.

Guzzo, R.A. y Shea, G.P. (1992). Group Performance and Intergroup Relations in Organizations. En M.D. Dunette y L.M. Hough (Eds.), Handbook of Industrial and Organization Psychology (pp. 269-313). Palo Alto, CA: Consulting Psychology Press.

Hackman, J. R. y Oldham, G. R. (1975). Development of the job diagnostic survey. Journal of Applied Psychology, 60(2), 159-170.

Humphrey, S. E., Nahrgang, J. D., \& Morgeson, F. P. (2007). Integrating motivational, social, and contextual work design features: A metaanalytic summary and theoretical extension of the work design literature. Journal of Applied Psychology, 92(5), 1332-1356.

Hofstede, G. (1980). Culture's consequences: International differences in work related values. Beverly Hills, CA: Sage.

Hofstede, G. (1993). Cultural constrains in management theories. Academy of Management Executive, 7, 81-94.

Huang, X. y Van de Vliert, E (2003). Comparing work behaviors across cultures: A cross-level approach using multilevel modelling. International Journal of Cross Cultural Management, 3 (2), 167-182.

Hyatt, D.E. y Ruddy, Th.M. (1997). An examination of the relationship between work group characteristics and performance: Once more into the breech. Personnel Psychology, 50(3), 553-585.

Ilgen, D.R., Mayor, D.A., Hollenbeck, J.R. y Sego, D.J. (1993). Team Research in the 1990s. En M.M Chemers y R. Ayman (Eds.), Leadership Theory and Research. Perspectives and Directions. San Diego: Academic Press.

Kilmann, R.H. y Saxton, M.J. (1983). The Kilmann-Saxton Cultura-Gap Survey. Pittsburg, PA.: Organizational Design Consultans. 
Kirkman, B.L. y Shapiro, D.L. (1997). The impact of cultural values on job satisfaction and organizational commitment in self-managing wok teams: the mediating role of employee resistance. Academy of Management Journal, 44(3), 557-569.

Kirkman, B.L. y Shapiro, D.L. (2001). The impact of cultural values on job satisfaction and organizational commitment in self-managing wok teams: the mediating role of employee resistance. Academy of Management Journal, 44(3), 557-569.

Klein, H. A., y Steele-Johnson, D. (2007). Training for multinational teamwork. In R. R. Hoffman (Ed.), Expertise out of context: Proceedings of the Sixth International Conference on Naturalistic Decision Making (pp. 473-506). New York: Lawrence Erlbaum.

Li, F., Li, Y. y Wang, E (2009). Task characteristics and team performance: the mediating effect of team member satisfaction. Social Behavior and Personality, 37(10), 1373-1382.

Musitu, G. y García, J.F. (2004). Consecuencias de la socialización familiar en la cultura española. Psicothema, 16(2), 288-293.

Newman, K.L. y Nollen, S.D. (1996). Culture and congruence: The fit between management practices and national culture. Journal of International Business Studies, 27, 753-779.

Nicholls, C.E., Lane, H.W., Brechu, M.B. (1999) Taking self-managed teams to Mexico. Academy of Management Executive, 13(3), 15-27.

Pavett, C. y Morris, T. (1995). Management styles within a multinational corporation: A five country comparative study. Human Relations, 48(10), 1171-1191.

Pelled, L. y Xin, K. (1997). Work values and their human resource management implications: A theoretical comparison of China, Mexico and the United States. Journal of Applied Management Studies, 6, 185-198.

Robert, C., Probst, T.M., Martocchio, J.J., Drasgow, F. y Lawler, J.L (2000). Empowerment and continuous improvement in the United States, Mexico, Poland and India: Predicting fit on the basis of the dimensions of power distance and individualism. Journal of Applied Psychology, 85(5), 643-658.

Ruiz, R., Sánchez, V. y Menesini, E. (2002). Violencia entre iguales y desconexión moral: un análisis transcultural. Psicothema, 14(Supl), 37-49.

Salas, E., Diaz, D., Klein, C. Burke, C. S., Stagl, K. C., Goodwin, G. F., \& Halpin, S. M. (2008). Does team training improve team performance? A meta-analysis. Human Factors, 50, 903-933.
Schwartz, S.H. (1992). Universals in the content and structure of values: Extensions and cross-cultural replications: Theorical advances and empirical tests in twenty countries. En M.P. Zanna (Ed.), Advances in Experimental Social Psychology (pp. 1-65). San Diego: Academic Press.

Shaw, J.D., Duffy, M.K. y Stark, E.M. (2000). Interdependence and preference for work group: Main and congruence effects on the satisfaction and the performance of group members. Journal of Management, 26(2), 259-279.

Smith, P.B., Dugan, S., Peterson, M.F. y Leung, K. (1998). Individualism: Collectivism and the handling of disagreement. A 23 country study. International Journal of Intercultural Relations, 22(3), 351-367.

Spector, P.E. (1985). Measurement of human service staff satisfaction: Development of the Job Satisfaction Survey. American Journal of Community Psychology, 13(6), 693-713.

Stewart, G. L. (2006). A meta-analytic review of relationships between team design features and team performance. Journal of Management, 32(1), 2955.

Strauch, B. (2010). Can Cultural Differences Lead to Accidents? Team Cultural Differences and Sociotechnical System Operations. Human Factors: The Journal of the Human Factors and Ergonomics Society, 52, 245-263.

Tannenbaum, S.I., Salas, E. y Cannon-Bowers, (1996). Promoting Team Effectiveness. En M.A. West (Ed.), Handbook of Work Group Psychology. Londres: John Wiley \& Sons.

Tschan, F. y Von Cranach, M. (1996). Group Task Structure, Processes and Outcomes. M.A. West (Ed.), Handbook of Work Group Psychology. Londres: John Wiley \& Sons.

Urien, B. (2001). Los grupos de trabajo y la efectividad grupal: un estudio en el sector de la automoción. Tesis Doctoral. Facultad de Psicología. Universidad Nacional de Educación a Distancia, Madrid.

Van Mierlo, H., Rutte, C. G., Kompier, M. A. J., y Doorewaard, H. A. (2005). Self-managing teamwork and psychological well-being: Review of a multilevel research domain. Group and Organization Management, 30(2), 211-235.

(Articulo recibido: 29-04-2010, revisado: 06-10-2011, aceptado: 16-11-2011) 